\title{
THE EFFICIENCY OF PULSE THERAPY WITH GLUCOCORTICOIDS IN INFLAMMATORY ORBITAL DISORDERS: CASE REPORT
}

\begin{abstract}
IOANA-CODRUȚA LEBĂDĂ $\check{1}^{1}$, MIHAELA STANCIU ${ }^{2}$
1,2 "Lucian Blaga" University of Sibiu, ${ }^{2}$ Sibiu County Emergency Clinical Hospital

Keywords: orbital Abstract: Orbital pseudotumor represents a benign inflammatory disorder of the orbit, with pseudotumor (OP), unspecified etiology, whose clinical presentation can mimic the ocular pathology of Basedow disease, graves' ophthalmopathy namely Graves' ophthalmopathy, the two of them representing two of the most common orbital (GO), glucocorticoids conditions. Imagistic methods and laboratory analyses can establish the diagnosis through orbital MRI images specific for the orbital pseudotumor, especially by dosage of the TSH receptor antibodies (TRAb) which will have increased values in Basedow disease with ocular damage. In both situations, the treatment is represented as a first therapeutic line by administration of corticotherapy in high dosage orally or intravenously, with favorably evolution, which is also observable in the case of our patient who was diagnosed with orbital pseudotumor based on MRI images, with associated thyroid dysfunction with negative TRAb, in which the pulse therapy with Solumedrol has determined the improvement of ocular symptoms and signs.
\end{abstract}

\section{INTRODUCTION}

Orbital pseudotumor (OP), also known as nonspecific orbital inflammation (NSOI) or idiopathic orbital inflammatory syndrome (IOIS) is a benign inflammatory dysfunction of the orbit, with unknown etiology.(1) OP represents approximately 8 to $11 \%$ of the orbital tumors and is the third most common orbital condition after Graves' ophthalmopathy and orbital lymphoma.(2)

From a clinical point of view, OP can manifest itself through a wide range of modifications depending on its location, affecting either one or several extraocular muscles (myositis), the lacrimal glands (dacryoadenitis), the sclera (scleritis), or damaging even the cavernous sinus (Tolosa-Hunt syndrome).(3) The diagnosis of the OP combines imagistic elements and laboratory data and is based on an exclusion diagnose after other orbital inflammatory pathologies are refuted; the treatment consists in administration of corticosteroids, external radiotherapy, chemotherapeutic agents, or surgery.(4,5)

Graves' ophthalmopathy or thyroid eye disease, the most common cause of uni or bilateral eye protrusion, is most often associated to hyperthyroidism in autoimmune thyroid dysfunction called Basedow disease but it should also be taken in consideration in euthyroid patients, with no apparent thyroid damage or in hypothyroid patients with chronic thyroiditis. $(6,7)$

Imaging scans such as CT and/or orbital MRI will identify possible existing orbital lesions, infiltrative processes, orbital idiopathic inflammation (pseudotumor) or other changes, while the TSH receptor antibodies (TRAb) analysis is useful in the positive diagnosis of ophthalmopathy, including in euthyroid patients.(8) Ocular imaging changes on MRI in Graves ophthalmopathy often involve bilateral damage with inflammation of the orbital muscle, without damage to the tendon as in the orbital pseudotumor, where we encounter the presence of a diffuse orbital mass with uveoscleral thickening, infiltration of the lacrimal gland, proptosis of the orbital muscle and of the tendon, with optic nerve thickening. $(8,9)$

The first choice of treatment in GO is intravenous corticosteroid therapy, a medication that has proven effective in orbital pseudotumor. $(8,9)$

\section{CASE PRESENTATION}

We present the case of a 40 -year-old male who presented to the Department of Endocrinology with bilateral ocular protrusion associated with diplopia and hyperlacrimation, progressively accentuated.

Objectively, the patient has a BMI of $29.35 \mathrm{~kg} / \mathrm{m}^{2}$, facies with bilateral exophthalmos, palpebral edemas, chemosis, motility disorders, bilateral positive Graefe sign, lagophthalmos, moderate EUGOGO score, NOSPECS score 3b; AP (arterial pressure) value of $130 / 90 \mathrm{mmHg}$, pulse of $84 \mathrm{bpm}$ (beats/minute), palpable thyroid gland, grade II goiter, firm consistency, irregular surface, mobile with deglutition, painless palpable deglutition, no neighboring adenopathy.

Hormonal dosage shows: initial $\mathrm{TSH}=0.01 \mu \mathrm{IU} / \mathrm{mL}$ (normal values $0.4-4$ ), and reaches the normal value of 3.37 $\mu \mathrm{IU} / \mathrm{mL}$ after treatment with antithyroid drugs (ATS), FT4 $=1.08$ $\mathrm{ng} / \mathrm{dl}$ (normal values $0.89-1.76$ ) (under treatment), $\mathrm{TRAb}=0.32$ $\mathrm{UI} / 1$ before charging (normal values $<1.58$ ), and $\mathrm{TRAb}=1.08$ $\mathrm{UI} / 1$ after repeated testing, thyroid peroxidase antibody $($ ATPO $)=85.67 \mathrm{UI} / \mathrm{mL}$ (normal values $0-34$ ), anti-Thyroglobulin antibodies $(\mathrm{ATG})=374.5 \mathrm{UI} / \mathrm{mL}$ (normal values $0-115$ ), IgG subclass $4=0.4290 \mathrm{~g} / \mathrm{L}$ (normal values $0.0300-2.0100$ ).

Thyroid ultrasound reveals an enlarged thyroid, with LDT volume of $9.8 \mathrm{~cm}^{3}$, isthmus $=3.3 \mathrm{~mm}$, LST volume of 5.3 $\mathrm{cm}^{3}$, nonhomogeneous nodular ecostructure of both thyroid lobes, nodule 1 at LDT level, with dimensions of 4.8/4.4 mm

${ }^{1}$ Corresponding author: Ioana Codruța Lebădă, Str. Prof. Dr. Ioan Moga, Nr. 1A, Sibiu, România, E-mail: codruta.racz@yahoo.com, Phone: +40722 614661

Article received on 26.10.2020 and accepted for publication on 15.12.2020 
transversal, 5.0/4.2 $\mathrm{mm}$ longitudinal, with central position, regular margins, vaguely delimitated and hypoechogenic, with microcalcifications, no vascularization, and nodule 2 at LTS level, with dimensions of $4.8 / 4.2 \mathrm{~mm}$ transversal, $5.8 / 5.0$ longitudinal, located on the posterior wall, with irregular margins, vaguely delimitated, hypoechogenic, with microcalcifications and no vascularization.

The ophthalmological evaluation highlights: visual acuity in the right eye (RE) of $0.7 \mathrm{fc}$, left eye (LE) of $1 \mathrm{fc}$; eye pressure $\mathrm{RE}=20 \mathrm{mmHg}, \mathrm{LE}=20 \mathrm{mmHg}$; fundus examination: contoured papillae, macula with reflex, vessels with increased parietal reflex, RE-limitation of sursumduction. Ophthalmometry shows RE $=22 \mathrm{~mm}, \mathrm{LE}=24 \mathrm{~mm}$, significant ocular protrusion on the left side.

It is initially performed a cranial CT (September, 2018), thus reaching to the conclusion of homogenous hypertrophy of inferior right muscle, RE with diameter of maximum $1.5 \mathrm{~cm}$ at the level of muscular corpus and minimum infiltration of retro orbital fat; the unilateral damage that includes the tendon inserts makes the appearance of ophthalmopathy less likely, thus inclining the diagnosis towards myositis/idiopathic orbital pseudotumor, yet without totally excluding the aspect of orbital lymphoma, which leads to the recommendation of an orbital MRI in order to fix the diagnosis. The MRI was performed subsequently with the result of thickening the inferior right muscle of the right eye up to $13 / 10$ $\mathrm{mm}$, uniformly, with myositis aspect. It is recommended that the investigations be completed by performing retrobulbar biopsy which is temporized up to present. The MRI exam is repeated in June 2019 (after specific treatment) with the following results: slight volume increase and discrete STIR hypersignal persistence in the lower right muscle on the right, discrete STIR hypersignal of the right lower and lateral muscles on the left, without changes in size.

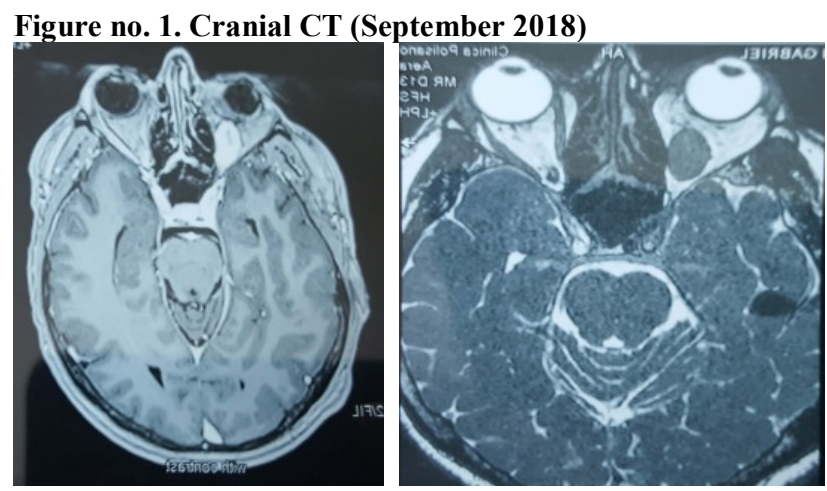

Figure no. 2. Cranial MRI at the moment of diagnosis (September 2018)

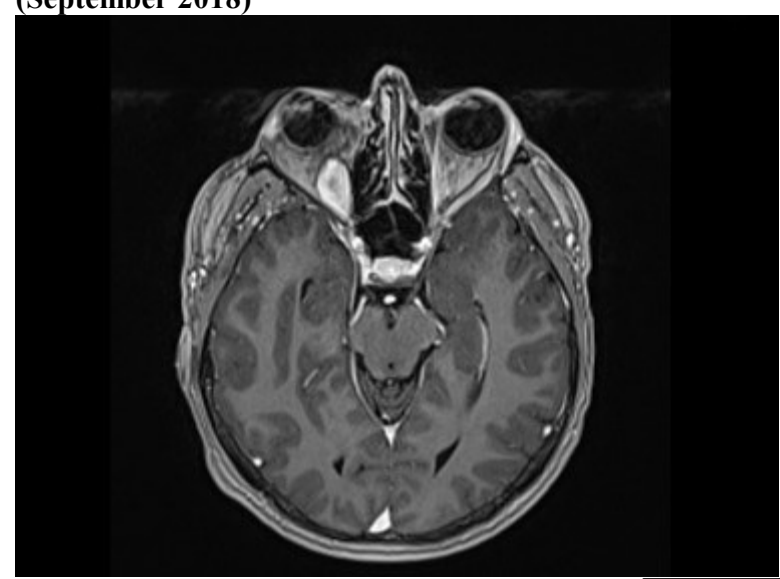

Based on the obtained clinical and paraclinical data the case was interpreted as Hashitoxicosis, toxic multinodular goiter, orbital pseudotumor of the right eye based on MRI investigation. Specific treatment with ATS was administrated to correct hyperthyroidism.

For the ocular symptoms, Medrol $32 \mathrm{mg} /$ day was initially administrated, with gradual reduction of dosage, but the treatment was poorly tolerated by the patient. No improvement of ocular symptoms was noticed, instead the patient presented associated clinical phenomena of iatrogenic Cushing syndrome due to the high dosage of glucocorticoids; therefore the oral therapy was stopped for three months, and the treatment was continued after this period with injectable Solumedrol in pulse therapy $1000 \mathrm{mg} /$ week for 8 weeks with favorable evolution and alleviation of symptomatology and clinical signs.

Figure no. 3. Cranial MRI after performing pulse therapy with Solumedrol RMN (June 2019)

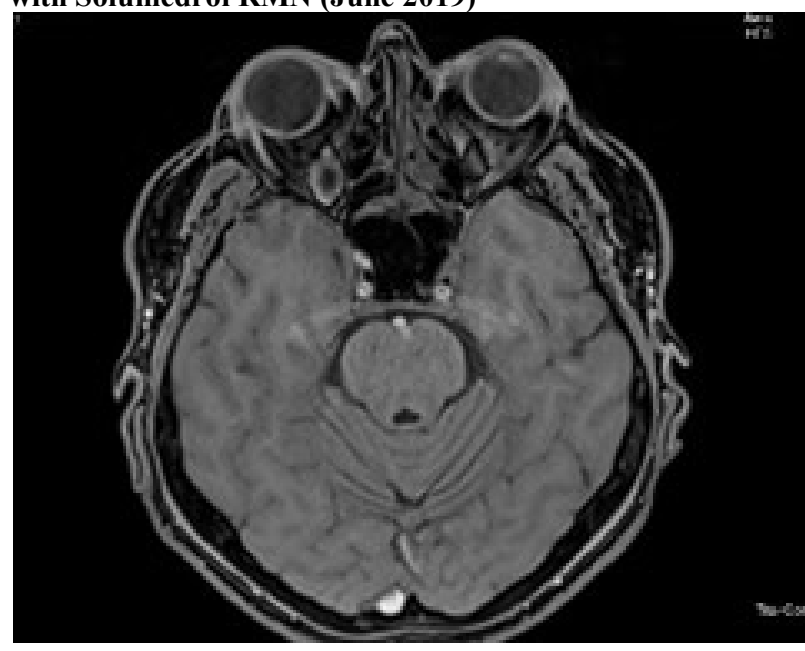

\section{DISCUSSIONS}

The orbital pseudotumour was first described by Gleason in 1903 and acknowledged as a clinical pathological identity in 1905 by Birch-Hirschfeld. It is defined as a benign inflammatory, space-replacing affection of the orbit, with no specific local or systemic etiology, which mostly occurs in adult population. $(1,10,11)$

Graves orbitopathy, the most common cause of exophthalmos, represents the most important extrathyroidal manifestation of Basedow disease, an immune affection which causes the inflammation and expansion of orbital muscles. (12)

Unilateral or bilateral exophthalmos, palpebral edemas, chemosis, diplopia leading even to compressive optical neuropathy with loss of vision in severe cases, all are signs and symptoms that can occur in GO, without these being specific, hence the difficulties of differential diagnosis in cases with such modifications.(13) Thus, other inflammatory, infectious, neoplastic, vascular or neuromuscular conditions must be taken into account, including the orbital pseudotumour which occurs in $40 \%$ of the cases.(14)

Although the GO diagnosis can easily be established in patients with hyperthyroidism and bilateral exophthalmos, it can be problematic in those with unilateral ocular damage, with normal thyroid function or hypothyroidism.(8) Imaging investigations such as CT and MRI will reveal all present modifications at orbital level, be them caused by an infiltrative process, an orbital idiopathic inflammation (pseudotumour) or other affections, while the dosage of thyroid-receptor antibodies (TRAb) with increased values will fix the diagnosis of Graves ophthalmopathy.(15) 


\section{CLINICAL ASPECTS}

Our patient presented in the unit care with bilateral ocular affection, more accentuated in the right eye, with onset of about three months, with hormonal dosage specific for hyperthyroidism in an autoimmune thyroid disease, but with repeated negative $T R A b$ values; the paraclinical investigations completed by the CT and MRI exams led the diagnosis towards an idiopathic orbital inflammation, orbital pseudotumour, by describing a unilateral condition that includes damage to the tendon inserts in the right eye. Similar cases have been described in specialized literature, including a case in our clinic published in 2016, referring to a patient with unilateral ocular modifications and thyroid dysfunction, initially diagnosed with GO, but later established as orbital pseudotumour, due to repeated negative TRAb values, MRI results, and orbital biopsy which confirmed the OP aspect.(16)

Systemic glucocorticoids are the main therapy in orbital pseudotumor, as well as the first choice of treatment in moderate and severe Graves ophthalmopathy, due to their antiinflammatory and immunosuppressive effects, with a prompt response and better tolerability when administered intravenously. $(9,17)$

Numerous studies on the efficiency of oral and intravenous corticotherapy have been performed over time and have proved the importance of this therapeutic method. A Turkish study published in 1996 shows the fact that 58.3\% (35 out of 60 patients) of the cases treated orally with high doses of corticosteroids had an optimal response to therapy.(18) Another study published in 2003 by Yuen and Rubin shows one more time the efficiency of glucocorticoids. Thus, 45 out of 65 patients diagnosed with OP were treated exclusively with corticosteroids, 8 of them underwent associated radiotherapy treatment, and the rest of them were treated with antiinflammatory non-steroids agents, $63 \%$ of the patients having optimal response to the administrated treatment, with complete remission of symptomatology in subsequent evaluations.(19) Our patient received initial treatment with oral glucocorticoids, then intravenously with a good response to this therapy, with relief of symptoms on reassessment.

\section{CONCLUSIONS}

As a conclusion, the hereby case demonstrates the importance of investigations in elucidating the diagnosis, our patient having thyroid dysfunction which - associated with ocular signs and symptoms - was interpreted in the context of Basedow disease. Subsequently, the diagnosis was invalidated by the repeated presence of negative TRAb values. Orbital MRI and $\mathrm{CT}$ has clarified the final diagnosis as right orbital pseudotumour associated with multinodular toxic goiter against the background of a chronic Hashimoto thyroiditis. The glucocorticoid treatment administrated orally and intravenously has significantly improved the symptoms.

The orbital pseudotumor is a relatively rare benign condition of the orbit that can clinically and radiologically mimic Graves orbitopathy, thus the differential diagnosis is compulsory in patients that present thyroid dysfunction with ocular damage, because - as presented in the previous case - the orbital pseudotumor can occur in association with thyroid pathology, other than Basedow-Graves disease.

\section{REFERENCES}

1. Kamili MA, G A, Dar IH, Dar SH, Wazir HS, Qureishi T. Orbital pseudotumor. Oman J Ophthalmol. 2009;2(2):9699.

2. Grouteau E, Chaix Y, Armbruster V, Lesueur L, Sevely A, Rubie H, Carriere JP. Acute orbital myositis and idiopathic inflammatory pseudotumor in children: three cases. Arch Pediatr. 1998;5:153-158.

3. Yeşiltaş YS, Gündüz AK. Idiopathic Orbital Inflammation: Review of Literature and New Advances. Middle East Afr J Ophthalmol. 2018;25(2):71-80.

4. Ferreira TA, Saraiva P, Genders SW, Buchem MV, Luyten GPM, Beenakker JW. CT and MR imaging of orbital inflammation. Neuroradiology. 2018;60(12):1253-1266.

5. Narla LD, Newman B, Spottswood SS, Narla S, Kolli R. Inflammatory Pseudotumor. Radiographics. 2003;23:7190720.

6. Bahn RS. Graves' ophthalmopathy. $\mathrm{N}$ Engl J Med. 2010;362(8):726-738.

7. Caminha LSC, Pinto ER, Medeiros de Sousa PA, Oliveira RA, Conceiçao FL, Vaisman M. Orbital pseudotumor: a differential diagnosis of Graves' ophthalmopathy. Arq Bras Endocrinol Metab. 2011;55(1):85-88.

8. Stan MN, Garrity JA, Bahn RS. The evaluation and treatment of graves ophthalmopathy. Med Clin North Am. 2012;96(2):311-328

9. Chaudhry IA, Shamsi FA, Arat YO, Riley FC. Orbital pseudotumor: distinct diagnostic features and management. Middle East Afr J Ophthalmol. 2008;15(1):17-27.

10. Gleason JE. Idiopathic Myositis involving the extraocular muscles. Opthalmol Rec. 1903;12:471-8.

11. Birch-Hirschfeld A. Zur diagnostic and pathologic der orbital tumoren. Ber Dtsch Opthalmol Ges. 1905;32:12735.

12. Bodu N, Jumani M, Wadhwa V, Bajaj G, Faas F. Not All Orbitopathy Is Graves': Discussion of Cases and Review of Literature. Front. Endocrinol. 2017;8(184):1-6.

13. Bahn RS, Heufelder AE. Pathogenesis of Graves' ophthalmopathy. N Engl J Med (1993) 329(20):1468-75.

14. Lacey B, Chang W, Rootman J. Nonthyroid causes of extraocular muscle disease. Surv Ophthalmol. 1999;44(3):187-213.

15. Khoo DH, Eng PH, Ho SC, et al. Graves' ophthalmopathy in the absence of elevated free thyroxine and triiodothyronine levels: prevalence, natural history, and thyrotropin receptor antibody levels. Thyroid. 2000;10:1093-1100.

16. Stanciu M, Popa FL, Totoian IG, Bera LG. Orbital pseudotumor can mimic Graves' ophthalmopathy. Acta Endocrinol (Buchar). 2016;12(3):344-348.

17. San Miguel I, Arenas M, Carmona R, Rutllan J, MedinaRivero F, Lara P. Review of the treatment of Graves' ophthalmopathy: The role of the new radiation techniques. Saudi J Ophthalmol. 2018;32(2):139-145.

18. Günalp I, Gündüz K, Yazar Z. Idiopathic orbital inflammatory disease. Acta Ophthalmol Scand. 1996 Apr;74(2):191-3.

19. Yuen SJ, Rubin PA. Idiopathic orbital inflammation: distribution, clinical features, and treatment outcome. Arch Ophthalmol. 2003 Apr;121(4):491-9. 